\title{
Criminologie
}

\section{Les praticiens de la criminologie au Québec}

\section{Rosette Gagnon et André Normandeau}

Volume 10, numéro 2, 1977

La criminologie au Québec

URI : https://id.erudit.org/iderudit/017072ar

DOI : https://doi.org/10.7202/017072ar

Aller au sommaire du numéro

Éditeur(s)

Les Presses de l'Université de Montréal

ISSN

0316-0041 (imprimé)

1492-1367 (numérique)

Découvrir la revue

Citer cet article

Gagnon, R. \& Normandeau, A. (1977). Les praticiens de la criminologie au Québec. Criminologie, 10(2), 39-54. https://doi.org/10.7202/017072ar d'utilisation que vous pouvez consulter en ligne.

https://apropos.erudit.org/fr/usagers/politique-dutilisation/ 


\section{INTRODUCTION}

Dans le cadre des travaux du Groupe de recherche sur la criminologie et les criminologues québécois (G.R.I.E. $n^{\circ} 3$, mai 1977), nous avons élaboré un questionnaire afin de réaliser un sondage auprès de tous les diplômés de l'́́cole de criminologie de l'Université de Montréal, de 1960 à 1976. Ce sondage était fait par la poste. Aussi, malgré les nombreux rappels, nous n'avons reçu que 149 questionnaires dûment remplis sur une population totale de 501 diplômés. Nous ne prétendons pas que notre «échantillon» soit pleinement représentatif et les résultats sont donc valables exclusivement pour ces 149 criminologues. Aussi, dans les pages qui suivent, le lecteur trouvera quelques statistiques intéressantes même si elles ne sont pas généralisables.

Nous présentons l'essentiel des résultats sous forme de : a) test , destiné aux lecteurs et visant à susciter leur intérêt ; les réponses à ce test sont tirées de nos résultats; b) résultats bruts au questionnaire (149 réponses); c) quelques tableaux statistiques chronologiques (1960-1976) *.

\section{A - TEST DESTINE AUX LECTEURS}

1 - Combien y a-t-il d'étudiants en criminologie ?

2-Quelle est la proportion d'étudiants pour qui le baccalauréat est une étape terminale d'études en criminologie ?

3 - Combien y a-t-il de diplômés de l'École de criminologie de l'Université de Montréal ?

4-Combien de diplômés ont obtenu à la fois le baccalauréat et la maîtrise en criminologie ?

5- Combien de diplômés ont obtenu à la fois la maîtrise et le doctorat en criminologie?

6-Combien de diplômés de l'École n'ont pas de baccalauréat de l’École ?

7 - Les diplômés de l'École de criminologie sont-ils surtout des femmes ou des hommes?

8 - Pour les cinq disciplines suivantes : sociologie, psychologie, droit, services social et criminel, pouvez-vous dire :

a) celle où l'on retrouve la proportion la plus grande de diplômés masculins ?

b) celle où l'on retrouve la plus faible proportion de diplômés féminins ?

c) comment se comparent la criminologie et la psychologie pour ce qui est des proportions de diplómés de chaque sexe.

- Cette présentation statistique est tiree du rapport de recherche no 3 du G.R.I.E. (Groupe de recherche sur la criminologie et les criminologues québecois) : R. Gagnon (André Normandeau, directeur), L'École de criminologie : les étudiants et les praticiens ", Ecole de criminologie, Universite de Montréal, 1977, 219 pages. 
9 - Quelles sont dans l'ordre les trois régions du Québec qui recrutent le plus grand nombre de criminologues diplômés de l'Université de Montréal ?

10 - Quels sont les cinq secteurs de travail où se dirigent le plus grand nombre de criminologues?

11 - Dans quelle proportion les criminologues sont-ils en contact direct avec des délinquants adultes ou juvéniles?

12 - Quel niveau de gouvernement emploie le plus de criminologues et dans quelle proportion?

13 - Quelle est la proportion des diplômés en criminologie qui, lors de leur inscription, n'avaient fait aucun autre choix ?

14 - Pour ceux qui ont mentionné d'autres choix d'orientation lors de leur inscription, quelles sont les trois disciplines les plus en demande?

15 - Quelle est la discipline de provenance đes diplômés de criminologie qui n'ont pas fait de baccalauréat à l'École ?

16 - Dans le choix de leurs cours optionnels, les diplômés de l'École disent avoir cherché à obtenir une formation surtout dans quel domaine?

17 - Ceux qui ont fait le baccalauréat en criminologie s'orientent-ils davantage vers une formation clinique proportionnellement à ceux qui ont complété un baccalauréat dans une autre discipline ?

18 - L'orientation poursuivie par un étudiant est-elle en relation quelconque avec le diplôme qu'il obtient ? (Bacc., Maîtrise, Doctorat)

19 - Pour l'ENSEMBLE du programme proposé par l'École, dans quelles proportions évaluez-vous le NOMBRE de cours offerts dans chacune des formations suivantes : Formation générale - Formation en recherche - Formation en clinique - Formation en sociologie et politique - Formation en a corrections * - Formation en administration?

20 - Pour les cours OPTIONNELS et pour les cours OBLIGATOIRES, dans quelles proportions évaluez-vous le NOMBRE de cours offerts dans chacune des formations ci-haut mentionnées?

21 -Dans le choix de leurs cours optionnels, dans quelles proportions les étudiants s'inscrivent à des cours faisant partie des formations suivantes : Formation en recherche - Formation en clinique Formation en sociologie et politique - Formation en * corrections * ?

22 - Quels sont les deux cours optionnels les plus \& populaires • à l'École de criminologie, c'est-à-dire ceux pour lesquels la proportion d'étudiants qui s'y inscrivent est la plus grande ? (Du moins pour la génération des diplômés 75-76.)

23 - De façon générale, le profil des cours optionnels choisis par un étudiant tout au long de son baccalauréat se compose de quels types de cours?

24-Quelle est la proportion des étudiants qui commencent un baccalauréat en criminologie et abandonnent pour une quelconque raison ?

25 - Y a-t-il beaucoup d'étudiants qui, bien qu'ayant complété un baccalauréat en criminologie, poursuivent ou recommencent leurs études dans une autre discipline? Vers quels domaines s'orientent-ils généralement ? 
26 - Y a-t-il beaucoup de diplômés qui, quoique diplômés de l'École de criminologie, abandonnent ce domaine pour travailler dans des secteurs qui n'ont rien à voir avec leurs études ?

\section{SOLUTIONS AU QUESTIONNAIRE}

1 - Il y avait 367 étudiants inscrits en 1976-1977.

2-Plus de $75 \%$.

$3-501$.

4 - Au moins 40 .

5 - Au moins 12.

6 - 90 điplômés soit $18 \%$.

7 - Masculin dans une proportion de $61 \%$.

8 - a) Droit ;

b) Service social ;

c) Criminologie : Masculin : $64 \%$; Féminin : $36 \%$; Psychologie : Masculin : $44 \%$; Féminin : $56 \%$; donc, des proportions inverses.

9-1. Montréal périphérique ;

2. Québec ;

3. Laurentides/Lanaudière.

$10-1$. Probation et protection des mineurs ;

2. Liberation conditionnelle ;

3. Probation adulte;

4. Institutions adultes ;

5. Recherche et enseignement ;

11 - Environ $75 \%$.

12 - Provincial avec plus de $45 \%$ des diplômés.

13 - Au moins $48 \%$.

14 - Psychologie : $36,5 \%$

Service social : $17,4 \%$

Droit : $11,1 \%$

15 - Sociologie pour $58 \%$ d'entre eux.

16 - Formation en criminologie clinique : $65,8 \%$ Formation en criminologie générale : $26,8 \%$

17 - Oui, cependant l'écart est faible. $67 \%$ de ceux qui ont fait le baccalaureat en criminologie et $59 \%$ pour les autres.

18 - Il semble que oui. Baccalauréat : Formation clinique : $72 \%$ - Formation générale : $28 \%$

Maîtrise : Formation clinique : $47 \%$ - Formation générale : $53 \%$ Doctorat : Formation clinique : NIL — Formation générale : $100 \%$

19 - Formation générale : $16,3 \%$

Formation en recherche : $11,6 \%$

Formation en clinique : $27,9 \%$

Formation en sociologie et politique : $18,6 \%$

Formation en * corrections * : 20,9\%

Formation en administration : $4,7 \%$. 
20-Formation générale :

Oblig. Option.

Formation en recherche :

$53,8 \%$

$23,1 \%$

$15,4 \%$

$6,7 \%$

Formation en clinique :

$7,7 \%$

$35,3 \%$

Formation en sociologie et politique :

$23,3 \%$

Formation en * corrections * :

Formation en administration :

- $\quad 30,0 \%$

$6,7 \%$

21 - Formation de recherche : $2,6 \%$ des inscriptions

Formation en clinique : $55,9 \%$ des inscriptions

Formation en sociologie et politique $: 14,8 \%$ des inscriptions

Formation en « corrections $»: 26,8 \%$ des inscriptions.

22-Clinique I et Technique d'entrevue I avec des taux d'inscription respectivement de $87,5 \%$ et de $82,1 \%$.

23 - Près de six cours de formation clinique ; environ 2,5 cours de formation en $\times$ corrections $*$; et à peine 1 cours dans la formation sociologie et politique.

24 - L'expérience a montré que de $25 \%$ à $33 \%$ des étudiants inscrits à la première anné du baccalauréat ne terminent pas le diplôme de premier cycle. Nous n'avons pas les proportions d'abandons et d'échecs. Surtout, nous ne savons pas le pourquoi du départ quand celui-ci est volontaire. Enfin, nous ne savons pas si ces étudiants s'orientent vers d'autres disciplines universitaires.

25 - Il nous est impossible d'Evaluer cette proportion en nombre. Toutefois, nous constatons qu'un nombre de plus en plus grand de diplômés s'inscrivent à la licence en droit ou encore au baccalauréat en administration.

26-Ce phénomène est réel mais encore une fois, nous n'en connaissons pas l'étendue. Par ailleurs, le baccalauréat en criminologie semble être polyvalent et multidisciplinaire à un point tel qu'il devient malaisé d'identifier ce qui est ou n'est pas un travail de nature criminologique...

\section{B. RÉSULTATS BRUTS AU QUESTIONNAIRE (149 điplômés)}

SEXE :

Masculin : $97(65,1)$

Féminin : $52(34,9)$

ÂE :

21 à 25 incl. : $49(32,9)$

26 à 30 incl. : $65(43,6)$

Plus de $30:(23,5)$

NATIONALITE :

Canadienne : 149 (100)

RÉGION DE NAISSANCE :

Bas St-Laurent/Gaspésie : $13(9,2)$

Saguenay/Lac St-Jean : $9(6,4)$

Québec : $27(19,1)$

Trois-Rivières : $9(6,4)$

Cantons de l'Est : $11(7,8)$

Montréal : $49(34,8)$ 
Laurentides/Lanaudière : $5(3,5)$

Montréal Sud : $7(5,0)$

Outaouais : $2(1,4)$

Nord-Ouest/Côte Nord : $2(1,4)$

Hors du Québec : $7(5,0)$

VOUS AVEZ VÉCU SURTOUT DANS UN MILIEU :

Urbain : $100(67,1)$

Rural : $24(16,1)$

Les deux également : $25(16,8)$

AVEC QUI VIVEZ-VOUS ?

Membre de la famille : $16(10,7)$

Époux, Epouse : $74(49,7)$

Ami(e) même sexe : $8(5,4)$

Ami(e) sexe différent : $25(16,8)$

Seul : $22(14,8)$

En groupe : $2(1,3)$

En communaute : $2(1,3)$

SCOLARITÉ DU PÉRE :

Universitaire : $24(16,3)$

Collégiale ou commerciale (12 à 14 ans) : $25(17,1)$

Secondaire (8 à 11 ans) : $49(33,3)$

Primaire (1 à 7 ans) : $49(33,3)$

OCCUPATION DU PËRE :

Professionnel ou semi-professionnel : $20(15,5)$

Administrateur/Gérant/Propriétaire : $42(32,6)$

Technicien/Ouvrier spécialisé : $14(10,9)$

Ouvrier semi-spécialisé : $22(17,1)$

Journalier/Secteur primaire : $20(15,5)$

Protection publique : $6(4,7)$

Bureau : $5(3,9)$

SCOLARITÉ DE LA MERE :

Universitaire : $5(3,4)$

Commerciale ou collégiale (12 à 14 ans) : $33(22,6)$

Secondaire (8 à 11 ans) : $51(34,9)$

Primaire (1 à 7 ans) : $57(39,0)$

OCCUPATION DE LA MÈRE :

Professionnelle ou semi-professionnelle : $16(11,3)$

Propriétaire : $1(0,7)$

Technicien/Ouvrier spécialisé : $3(2,1)$

Ouvrier semi-spécialisé : $3(2,1)$

Journalier : $1(0,7)$

Bureau : $12(8,5)$

Emploi de service : $3(2,1)$

Sans emploi : $102(72,3)$

AVEZ-VOUS VOTRE BACCAI.AUREAT EN CRIMINOLOGIE ?

Oxi : $122(81,9)$

Non : $27(18,1)$ 
QUEL DIPLÓME DETENEZ-VOUS ?

Baccalauréat : $107(71,8)$

Maîtrise : $37(24,9)$

Doctorat : $5(3,4)$

EN QUELLE ANNEE AVEZ-VOUS EU CE DIPLOME ?

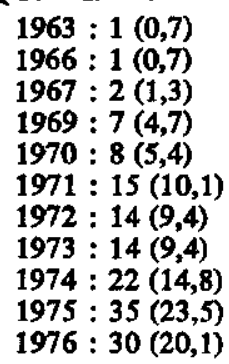

AVEZ-VOUS D'AUTRES DIPLOMES ?

Oui : $45(30,2)$

Non : $104(69,8)$

SI OUI, QUELS DIPLÔMES ?

Discipline d'orientation * clinique : : $6(13,6)^{\text {1 }}$

Discipline en sciences sociales : $16(36,4)$

Droit : $4(9,1)$

Administration : $2(4,5)$

Philosophie/Théologie : $4(9,5)$

Autres : $12(27,3)$

LORS DE VOTRE INSCRIPTION EN CRIMINOLOGIE, AVIEZ-VOUS FAIT D'AUTRES CHOIX ?

Oui : $70(47,0)$

Non : $79(53,0)$

SI OUI, QUELS CHOIX ?

Discipline d'orientation = clinique s: $38(55,1)^{2}$

Discipline en sciences sociales : $16(23,2)$

Droit : $8(11,6)$

Sciences exactes : $7(10,1)$

DANS LE CHOIX DE VOS COURS OPTIONNELS À L'ÉCOLE, VOUS AVEZ CHERCHÉ À AVOIR UNE FORMATION SURTOUT EN :

Clinique : $98(65,8)$

Recherche : $5(3,4)$

Administration : $1(0,7)$

Sciences policières : $2(1,3)$

1. Orientation eclinique, : Psychologie : 4; Drogue et alcoolisme : 2 . Discipline en sciences sociales : Sociologie : 13; Anthropologie : 2 ; non précise : 1 . Autres : B.A.; Programmation; Audiologie.

2. Orientation ecliniques: Psychologie : 23 ; Service social : 11 ; Psychoeducation : 4. Discipline en sciences sociales : Sociologie : 4; Relations Industrielles : 4; Economie : 3 ; Anthropologie : 3 ; Polltique : 1; Démographio: 1 . 
Criminologie critique : $3(2,0)$

Criminologie générale : $6(4,0)$

Combinaison de plusieurs : $34(22,8)$

ENDROITS DES STAGES 1 ET 2 :

Centres de recherche :

Institutions pour adultes :

STAGE 1 STAGE 2

Centres d'accueil :

Centres hospitaliers :

Centres des services sociaux ${ }^{3}$ :

Probation juvénile :

Probation adulte :

Liberation conditionnelle :

Police :

Centres résidentiels commun. :

Milieu scolaire :

Autres :

Pas fait de stage :

$9(6,0) \quad 10(6,7)$

$32(21,5) \quad 11(7,8)$

$26(17,4) \quad 16(10,7)$

$8(5,4) \quad 4(2,7)$

$3(2,0) \quad 11(7,4)$

$10(6,7) \quad 7(4,7)$

$9(6,0) \quad 14(9,4)$

$9(6,0) \quad 19(12,8)$

$11(7,4) \quad 6(4,0)$

$1(0,6) \quad 2(1,3)$

$6(4,0) \quad 9(6,0)$

$6(4,0) \quad 13(8,7)$

$19(12,8) \quad 27(18,1)$

Róle Jout AU COURS DU STAGE :

Recherche :

Clinique :

Administration :

Observation :

Réalisation d'un projet :

STAGE 1 STAGE 2

$12(9,2) \quad 21(17,2)$

$88(67,7) \quad 88(72,1)$

$6(4,6) \quad 7(5,7)$

$24(18,5) \quad 4(3,3)$

- $2(1,6)$

\section{REGIONS DES STAGES :}

Bas St-Laurent/Gaspésie :

STAGE 1 STAGE 2

Saguenay/Lac St-Jean :

Québec :

Trois-Rivières :

Cantons de l'Est :

Montréal :

Laurentides/Lanaudière :

- $3(2,5)$

$6 \overline{(4,0)} \quad 1(0,7)$

$6(4,6) \quad 8(6,6)$

$2(1,5) \quad 1(0,8)$

$2(1,5) \quad 1(0,8)$

$91(70,0) \quad 84(68,9)$

Montréal Sud :

$15(11,5)$

$12(9,2)$

$10(8,2)$

Outaouais :

Nord-Ouest/Côte Nord :

$1(0,8)$

$1(0,8)$

$10(8,2)$

Hors Québec :

$1(0,8)$

$3(2,5)$

LA CRIMINOLOGIE TELLE QU'ELLE VOUS A ETTE ENSEIGNEE A L'ÉCOLE CORRESPONDAIT-ELLE À L'IDEE QUE VOUS EN AVIEZ LORS DE VOTRE INSCRIPTION ?

Beaucoup : $45(30,2)$

Passablement : $82(55,0)$

Aucunement : $22(14,8)$

3. Nous n'avons pas inclus ici tous les aervices du C.S.S. : la probation juvenile, par exemple, est considéréte séparément. 
COMMENT QUALIFIERIEZ-VOUS GLOBALEMENT LA FORMATION CRIMINOLOGIQUE QUE VOUS AVEZ REÇUE A L'ÉCOLE ?

Très satisfaisante : $27(\mathbf{1 8 , 1 )}$

Satisfaisante : $82(55,0)$

Insatisfaisante : $40(26,8)$

EMPLOIS OCCUPES DEPUIS LE DERNIER DIPLÔME 4 :

Centres de recherche :

Institutions pour adultes :

Centres d'accueil :

Centres hospitaliers :

C.S.S. 5 :

Probation juvénile :

Probation adulte :

Libération conditionnelle

Milieu scolaire :

Police :

$\begin{array}{cccc}A C T U E L & 3 & 2 & 1 \\ 9(6,0) & 6(8,0) & 2(7,1) & 2(20,0) \\ 12(8,1) & 8(10,7) & 4(14,3) & 1(10,0) \\ 7(4,7) & 13(17,3) & 2(7,1) & - \\ 3(2,0) & - & 2(7,1) & 1(10,0) \\ 17(11,4) & 8(10,4) & 2(7,1) & 2(20,0) \\ 9(6,0) & 1(1,3) & 2(7,1) & - \\ 28(18,8) & 5(6,7) & 4(14,3) & - \\ 14(9,4) & 12(16,0) & 3(10,7) & - \\ 3(2,0) & 3(4,0) & 1(3,6) & - \\ 4(2,7) & - & - & - \\ 12(8,1) & 7(9,3) & 3(10,7) & 2(20,0) \\ 3(2,0) & 2(2,7) & - & - \\ 12(8,1) & 10(13,1) & 3(10,7) & 2(20,0) \\ 2(1,3) & - & - & - \\ 6(4,0) & - & - & - \\ 8(5,4) & - & - & -\end{array}$

$\begin{array}{lrr}\text { Maisons d'enseignement : } & 12(8,1) & 7(9,3) \\ \text { M.A.S. : } & 3(2,0) & 2(2,7 \\ \text { Autres : } & 12(8,1) & 10(13,1) \\ \text { Travail non criminologique : } & 2(1,3) & \\ \text { Chômage : } & 6(4,0) & - \\ \text { Etudiant : } & 8(5,4) & \\ \text { RÔLE JOUÉ DANS CHACUN DES EMPLOIS : }\end{array}$

Recherche :

Clinique :

Administration :

Enseignement :

$\begin{array}{lccc}A C T U E L & 3 & 2 & 1 \\ 13(9,8) & 9(12,0) & 3(10,3) & 1(12,5) \\ 77(58,4) & 48(64,0) & 18(62,1) & 4(50,0) \\ 31(23,6) & 11(14,7) & 5(17,2) & 1(12,5) \\ 11(8,3) & 7(9,3) & 3(10,3) & 2(25,0)\end{array}$

RÉGIONS DES EMPLOIS :

$\begin{array}{lcccc} & \text { ACTUEL } & 3 & 2 & 1 \\ \text { Bas St-Laurent/Gaspésie : } & 5(3,7) & 1(1,3) & 2(6,9) & - \\ \text { Saguenay/Lac St-Jean : } & 2(1,5) & - & 1(3,4) & - \\ \text { Québec : } & 20(15,0) & 6(8,0) & - & 1(12,5) \\ \text { Trois-Rivières : } & 4(3,0) & 2(2,7) & - & - \\ \text { Cantons de l'Est : } & 2(1,5) & - & - & - \\ \text { Montréal : } & 64(48,4) & 44(58,7) & 19(65,5) & 6(75,0) \\ \text { Laurentides/Lanaudière : } & 13(9,7) & 9(12,0) & 4(13,8) & - \\ \text { Montréal Sud : } & 14(10,4) & 5(6,7) & 1(3,4) & - \\ \text { Outaouais : } & 2(1,5) & 1(1,3) & 1(3,4) & - \\ \text { Nord-Ouest/Côte Nord : } & 1(0,7) & 2(2,7) & 1(3,4) & - \\ \text { Hors Québec : } & 6(4,5) & 5(6,7) & - & 1(12,5)\end{array}$

4. 1 correspond au premier emploi occupé, 2 au second, 3 au troisieme et e actuel , au travail présent du diplómé.

5. Nous n'avons pas inclus ici tous les services du C.S.S. : la probation juvénile, par exemple, est considéré séparément. 
TRAITEMENT ANNUEL POUR LES EMPLOIS :

$\begin{array}{lcccc} & \text { ACTUEL } & 3 & 2 & 1 \\ \text { Moins de } 11000: & 27(21,1) & 30(44,8) & 13(52,0) & 6(85,7) \\ \text { De } 11000 \text { à } 13999: & 35(27,3) & 19(28,4) & 7(28,0) & 1(14,3) \\ \text { De } 14000 \text { à } 16999: & 26(20,3) & 15(22,4) & 4(16,0) & - \\ 17000 \text { et plus : } & 40(31,3) & 3(4,5) & 1(4,0) & -\end{array}$

COMBIEN DE TEMPS A CET ENDROIT ?

Moins de 6 mois :

\begin{tabular}{lccc} 
ACTUEL & \multicolumn{1}{c}{3} & 2 & 1 \\
$27(20,6)$ & $18(24,0)$ & $4(13,8)$ & $1(12,5)$ \\
$21(16,0)$ & $12(16,0)$ & $7(24,1)$ & $3(37,5)$ \\
$16(12,2)$ & $15(20,0)$ & $2(6,9)$ & - \\
$12(9,2)$ & $12(16,0)$ & $4(13,8)$ & $2(25,0)$ \\
$19(14,5)$ & $6(8,0)$ & $6(20,7)$ & $2(25,0)$ \\
$36(27,5)$ & $12(16,0)$ & $6(20,7)$ & -
\end{tabular}

RAISONS DU DEPART :

Salaire et conditions de travail :

$\begin{array}{ccc}3 & 2 & 1 \\ 3(4,2) & 1(3,6) & -\end{array}$

Changement d'orientation, de préférences :

$21(29,2) \quad 14(50,0) \quad 1(16,7)$

Insatisfaction quant au type de travail : $\quad 16(22,2) \quad 4(14,3) \quad 1(16,7)$

Manque de travail, fin de contrat: $\quad 10(13,9) \quad 4(14,3) \quad 2(33,3)$

Pour avoir une promotion :

$12(16,7) \quad 2(7,1) \quad 2(33,3)$

Pour cause de retour aux études :

$4(5,5)$

$3(10,7)$

-

NOMBRE D'EMPLOIS OCCUPES DEPUIS LE DIPLÓME :

Aucun : $12(8,3)$

Un : $64(43,0)$

Deux : $45(30,2)$

Trois : $28(18,8)$

CONSIDÉREZ-VOUS QU'IL EXISTE UN LIEN

ENTRE LA FORMATION QUE VOUS AVEZ RECUE A L'ECOLE

ET LA PRATIQUE QUE VOUS EXERCEZ MAINTENANT ?

Très net : $42(31,8)$

Passable : $58(43,9)$

Aucun : $32(24,2)$

DANS VOTRE MILIEU DE PRATIQUE ACTUEL,

TRAVAILLEZ-VOUS AVEC DES PROFESSIONNELS

D'UNE AUTRE DISCIPLINE QUE LA CRIMINOLOGIE ?

Oui : $123(92,5)$

Non : $10(7,5)$

SI OUI, CEUX-CI EXERCENT :

Le même travail que vous : $73(60,3)$

Un travail semblable : $31(25,6)$

Un travail différent : $17(14,0)$ mềme endroit.

6. Dans co cas il s'agit du temps depuis lequel le répondant travaille au 
DANS VOTRE MILIEU DE TRAVAIL ACTUEL,

Y A-T-IL DES PERSONNES SANS FORMATION UNIVERSITAIRE QUI EXERCENT LA MEME FONCTION QUE VOUS ?

Oui : $49(37,1)$

Non : $83(62,9)$

SI OUI, LEUR RENDEMENT EST :

Supérieur au vôtre : $1(2,1)$

Égal au vôtre : $35(72,9)$

Inférieur au vôtre : $12(25,0)$

CONSIDEREZ-VOUS QUE VOTRE MILIEU DE TRAVAIL SERAIT PLUS EFFICACE S'IL NE COMPORTAIT QUE DES

CRIMINOLOGUES ?

Oui : $6(4,6)$

Non : $113(86,9)$

Indécis : $11(8,5)$

VOTRE DIRECTEUR EST-IL CRIMINOLOGUE ?

Oui : $47(35,1)$

Non : $84(62,7)$

Je suis directeur : $3(2,2)$

DANS LE CADRE DE VOTRE PRATIQUE QUOTIDIENNE, VOTRE TRAVAIL CONSISTE SURTOUT $\AA$ :

$1^{\text {er }}$ choix $2^{\mathrm{e}}$ choix $3^{\mathrm{e}}$ choix

Diagnostiquer, plan de traitement, traiter :

Faire de la recherche :

$76(57,1) \quad 77(58,3) \quad 67(53,2)$

Administrer :

$11(8,3) \quad 11(8,3) \quad 3(2,4)$

Enseigner :

$20(15,0) \quad 18(13,6) \quad 16(12,7)$

Autres :

$15(11,3) \quad 19(14,4) \quad 24(19,0)$

$\begin{array}{lll}11(8,3) & 7(5,3) & 16(12,7)\end{array}$

IDÉALEMENT, VOTRE TRAVAIL DEVRAIT CONSISTER SURTOUT $\bar{A}$ :

Diagnostiquer, plan de traitement,

$1^{\text {ex }}$ choix $2^{\mathrm{e}}$ choix $3^{\mathrm{e}}$ choix traiter :

Faire de la recherche :

$72(54,1) \quad 72(54,1) \quad 57(44,9)$

Administrer :

Enseigner :

Autres :

$7(5,3) \quad 17(12,8) \quad 5(3,9)$

$19(14,3) \quad 12(9,1) \quad 10(7,9)$

$16(12,0) \quad 13(9,8) \quad 13(10,2)$

$19(14,3) \quad 19(14,3) \quad 42(33,1)$

ENVISAGEZ-VOUS DE QUITTER VOTRE EMPLOI

À PLUS OU MOINS BREVE ÉCHÉANCE ?

Oui : $37(27,6)$

Non : $75(56,0)$

Indécis : $22(16,4)$

SI OUI OU INDÉCIS, POURQUOI ?

Salaire et conditions de travail : $6(10,5)$

Changement d'orientation, de préférence : $21(36,8)$

Insatisfaction quant au type de travail : $16(28,1)$ 
Manque de travail, fin du contrat : $4(7,0)$

Pour avoir une promotion : $1(1,8)$

Pour cause de retour aux études : $3(5,3)$

Autres : $6(10,5)$

FAITES-VOUS PARTIE DE L'A.P.C.Q. (Association professionnelle des criminologues du Québec) ?

$$
\text { Oui : } 74(49,7) \text { - Non : } 75(50,3)
$$

\section{SI NON, POURQUOI ?}

Je ne Ia connais pas : $14(19,7)$

Je n'en vois pas l'utilité : $32(45,1)$

Je n'en ai pas le temps : $4(5,6)$

Ca ne m'intéresse pas : $8(11,3)$

Par négligence de payer ma cotisation : $13(18,3)$

\section{UNE CORPORATION DE CRIMINOLOGUES EST-ELLE}

SOUHAITABLE ?

Oui : $85(57,4)$

Non : $28(18,9)$

Indécis : $35(23,6)$

QUELS DEVRAIENT ÊTRE LES OBJECTIFS D'UNE

ASSOCIATION $\square$ OU D'UNE CORPORATION $\square$ ?

[Association : $48(32,2)$ - Corporation : $65(43,6)$ -

non précisé : $36(24,2)]$

$\begin{array}{lccccc} & 1 \text { er } & 20 & 3 \mathrm{e} & 4^{\mathrm{e}} & 5^{\mathrm{e}} \mathrm{z} \\ \begin{array}{c}\text { Protection de } \\ \text { la profession : }\end{array} & 51(42,1) & 46(41,1) & 30(32,6) & 16(30,0) & 1(6,3) \\ \begin{array}{c}\text { Protection } \\ \text { des membres : }\end{array} & 67(55,4) & 61(54,5) & 50(54,3) & 30(57,7) & 10(62,5) \\ \begin{array}{c}\text { Protection } \\ \text { du public : }\end{array} & 3(2,5) & 5(4,5) & 12(13,0) & 6(11,5) & 5(31,3)\end{array}$

ÊTES-VOUS INFORMÉ DE CE QUI SE PASSE À L'ÉCOLE

DE CRIMINOLOGIE ?

Aucunement : $49(32,9)$

Rarement : $58(38,9)$

Souvent : $42(28,2)$

SI OUT, COMMENT ?

Je m'y rends moi-même, j'y travaille : $38(31,9)^{8}$

Par ses publications : $11(9,2)$

Par des stagiaires : $42(35,3)$

Par des amis étudiants : $26(21,8)$

Par des professeurs : $28(23,5)$

Par ouï-dire : $37(31,1)$

Par l'A.P.C.Q. : $43(36,1)$

Par les média : $24(20,2)$

Par des colloques, congrès : $33(27,7)$

7. Il ne s'agit pas là d'un ordre de priorité.

8. Les pourcentages ne s'additionnent pas. Les répondants pouvalent mentionner plus d'une alternative. 
ETTES-VOUS INFORME DE CE QUI SE PASSE DANS LE SECTEUR DE LA CRIMINOLOGIE EN GÉNERAL, EN DEHORS DE L'ECOLE?

Aucunement : $29(19,5)$

Rarement : $54(36,2)$

Tres au courant : $66(44,3)$

\section{SI OUI, COMMENT ?}

Par des publications : $69(51,9)$

Par l'A.P.C.Q. : $44(33,1)$

Par des amis, confrères : $80(60,2)$

Par ouï-dire : $18(13,5)$

Par des réunions, congrès, rencontres : $50(37,6)$

Par les média : $62(46,6)$

Par des stagiaires : $26(19,5)$ 
NOMBRE D'ETUDIANTS INSCRITS À L'ECOLE DE CRIMINOLOGIE

\begin{tabular}{|c|c|c|c|c|c|c|c|c|c|c|c|c|c|c|c|c|c|}
\hline & 1960 & 1961 & 1962 & 1963 & 1964 & 1965 & 1966 & 1967 & 1968 & 1969 & 1970 & 1971 & 1972 & 1973 & 1974 & 1975 & 1976 \\
\hline Bac. I & - & - & - & - & - & - & - & 62 & 104 & 52 & 57 & 67 & 108 & 111 & 105 & 98 & 91 \\
\hline Bac. II & - & 一 & - & - & 一 & - & - & - & 44 & 79 & 42 & 57 & 64 & 81 & 85 & 84 & 80 \\
\hline Bac. III & - & - & - & - & - & - & - & - & - & 43 & 82 & 42 & 42 & 56 & 60 & 74 & 77 \\
\hline & & & & & & & & 62 & 148 & 174 & 181 & 166 & 214 & 248 & 250 & 256 & 248 \\
\hline Maîtrise * & 13 & 16 & 27 & 27 & 30 & 44 & 52 & 48 & 55 & 61 & 71 & 50 & 87 & 103 & 91 & 121 & 97 \\
\hline Ph.D. * & - & - & - & 一 & 3 & 10 & 10 & 15 & 26 & 31 & 25 & 23 & 25 & 21 & 20 & 21 & 22 \\
\hline TOTAL & 13 & 16 & 27 & 27 & 33 & 54 & 62 & 125 & 229 & 266 & 277 & 239 & 326 & 372 & 361 & 398 & 367 \\
\hline
\end{tabular}

- Les chiffres de maitrise et doctorat comprennent les etudiants actifs et ceux qui sont en rédaction de mémoire et de thèso. 


\section{NOMBRE D'ETUDIANTS INSCRITS}

A L'ECOLE DE CRIMINOLOGIE

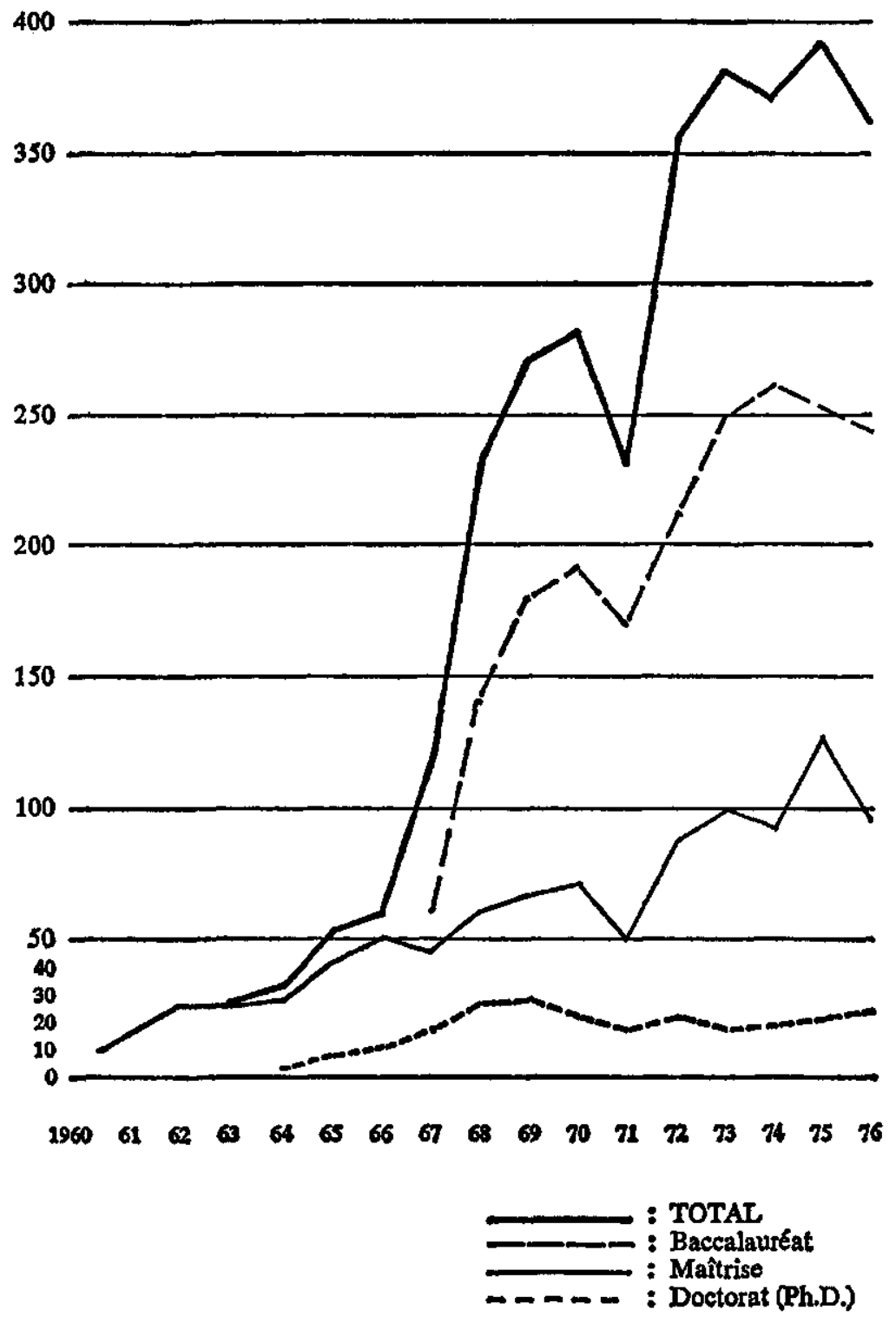


Appendice $C$ NOMBRE DE PROFESSEURS A LECOLE DE CRIMINOLOGIE

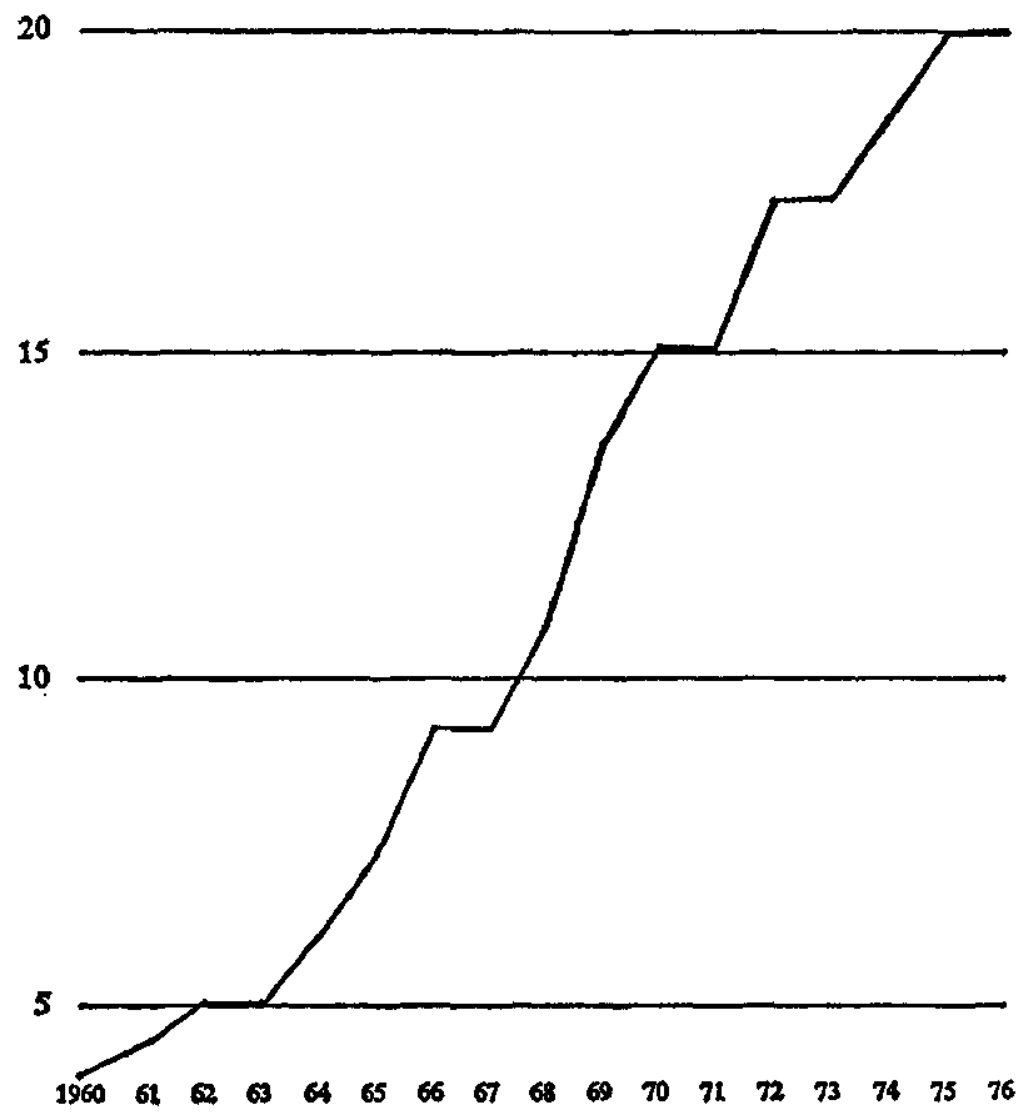


Appendice $D$

DIPLOOMES DECERNES PAR L'ECOLE * DE CRIMINOLOGIE DE L'UNIVERSITÉ DE MONTREAL (1963-1976)

\begin{tabular}{ccrcr}
\hline Année & Baccalauréat & Maîtrise & Doctorat & Total \\
\hline 1963 & & $3(100)$ & & 3 \\
1964 & & $3(100)$ & & 3 \\
1965 & & $2(100)$ & & 2 \\
1966 & & $7(100)$ & & 7 \\
1967 & & $10(100)$ & & 10 \\
1968 & & $7(87,5)$ & $1(12,5)$ & 8 \\
1969 & & $10(90,9)$ & $1(9,1)$ & 11 \\
1970 & $39(73,6)$ & $9(17,0)$ & $5(9,4)$ & 53 \\
1971 & $76(85,4)$ & $10(11,2)$ & $3(3,4)$ & 89 \\
1972 & $40(80,0)$ & $9(18,0)$ & $1(2,0)$ & 50 \\
1973 & $48(85,7)$ & $7(12,5)$ & $1(1,8)$ & 56 \\
1974 & $50(75,8)$ & $14(21,2)$ & $2(3,0)$ & 66 \\
1975 & $78(75,7)$ & $23(22,3)$ & $2(1,9)$ & 103 \\
1976 & $80(87,0)$ & $11(12,0)$ & $1(1,0)$ & 92 \\
Total : & $411(74,3)$ & $125(22,6)$ & $17(3,1)$ & 553 \\
\hline
\end{tabular}

- L'Ecole a accordé 553 diplames à 501 personnes, ce qui signifie que 52 d'entre elles possèdent deux diplömes de l'École. 